cerebral edema developed immediately after pneumoencephalography an died. Autopsy showed cerebral edema, especially marked in the cerebellum plexus choriodideus was normal and no space occupying lesion was seet In the two autopsied cases, no sinus thrombosis was observed.

The etiology of high intracranial pressure in such cases is obscure an probably various. Prognosis is often favorable and symptoms disappea spontaneously. In 2 lethal cases, cause of death was considered iatrogenic In management of intracranial hypetension without focal signs, just watch ing the course in parallel with careful control of water metabolism is re commended. If choked disc persists, subtemporal decompression is th treatment of choice.

\title{
61. The Study on the Effects of FRUCTON-M, Mixture of $45 \%$ Fructose Solution and 15\% Mannitol Solution, for the Reduction of Increased Intracranial Pressure
}

\author{
Yuji Mryazaki, Shinshyo Matsumoto and Masayuki Takeda \\ Dept. of Neurosurgery, Sapporo Medical College
}

Osmotherapeutic agents are indispensable in the field of neurosurgery In the 22nd and 23rd Annual Meeting of Japan Neurosurgical Society, wr had reported the effects of hypertonic mannitol solution which is able ts replace with hypertonic urea solution, and emphasized hypertonic mannito solution is an ideal one, except great amount of solution has to be infusec into the circulation system by application. This disadvantage of hypertonic mannitol solution was the point which should be improved in near future.

The authors had developed new osmotherapeutic agent, FRUCTON-M which contains $45 \%$ fructose solution and $15 \%$ mannitol solution with ratt of 3:1 after basic research of osmotherapeutic effect of $50 \%$ fructose solution

For the sake of usage, FRUCTON-M was used at a rate of $7 \mathrm{cc} / \mathrm{kg}$ for 30-40 minutes by intravenous instillation.

The cerebrospinal fluid pressure drop rate was $41.5-95.2 \%$, and $71.4 \%$ the average.

Reducing action lasted 120-360 minutes, and 246 minutes on the average

The secondary rebound overshoot was observed in $9.5 \%$ of all cases, and completely same frequency and grade with hypertonic mannitol solution. 